\title{
Decreased CD117 expression in hypopigmented mycosis fungoides correlates with hypomelanosis: lessons learned from vitiligo
}

\author{
Zeba N Singh ${ }^{1}$, Maria S Tretiakova ${ }^{1}$, Christopher R Shea ${ }^{2}$ and Vesna M Petronic-Rosic ${ }^{2}$ \\ ${ }^{1}$ Department of Pathology, University of Chicago Hospitals, Chicago, IL, USA and ${ }^{2}$ Section of Dermatology, \\ Department of Medicine, University of Chicago Hospitals, Chicago, IL, USA
}

\begin{abstract}
Hypopigmented mycosis fungoides is an uncommon clinical variant of cutaneous T-cell lymphoma. We hypothesized that hypomelanosis in hypopigmented mycosis fungoides may have a similar mechanism as in vitiligo, a condition in which it is believed that alterations in expression of CD117 (stem cell factor receptor/KIT protein) on epidermal melanocytes and abnormal interactions between melanocytes and surrounding keratinocytes may play a pathogenic role. To test the hypothesis that similar mechanisms might also explain hypopigmentation in hypopigmented mycosis fungoides, skin specimens from five cases each of hypopigmented mycosis fungoides and vitiligo were studied immunohistochemically for immunophenotype of the infiltrating cells, CD117 (expressed by epidermal melanocytes), and pan melanoma cocktail of antigens (gp100, tyrosinase, and MART-1) expression; cases of conventional mycosis fungoides and normal skin were studied in parallel as controls. Our findings confirm a predominance of CD8 + neoplastic T cells in hypopigmented mycosis fungoides. Similarly, the epidermal lymphocytic infiltrate in vitiligo was also composed of CD8 + cytotoxic $\mathrm{T}$ cells, in contrast to an epidermal infiltrate composed of CD4 $+\mathrm{T}$ cells in conventional mycosis fungoides. The average number of epidermal CD117 expressing cells followed the same pattern of decreased expression in hypopigmented mycosis fungoides as in vitiligo, whereas the levels in conventional mycosis fungoides were higher, and similar to that observed in normal skin. Furthermore, a decreased number of melanocytes per high-power field of the length of the biopsy was present in hypopigmented mycosis fungoides and vitiligo, as compared with either conventional mycosis fungoides or normal skin, suggesting a correlation between decreased expression of CD117 and decreased number of melanocytes. We propose that decreased expression of CD117 and its downstream events in melanocytes may be initiated by cytotoxic effects of melanosomal-antigen-specific CD8 + neoplastic T lymphocytes, resulting in destabilization of CD117 and leading to dysfunction and/or loss of melanocytes in the epidermis of hypopigmented mycosis fungoides. Modern Pathology (2006) 19, 1255-1260. doi:10.1038/modpathol.3800644; published online 16 June 2006
\end{abstract}

Keywords: hypopigmented mycosis fungoides; vitiligo; pathogenesis; melanosomal antigens; CD117; KIT

Hypopigmented mycosis fungoides is a variant of cutaneous T-cell lymphoma more commonly seen in dark-skinned individuals. ${ }^{1-4}$ The natural history of hypopigmented mycosis fungoides is similar to that of conventional mycosis fungoides, although the

Correspondence: Dr V Petronic-Rosic, MD, MSc, Section of Dermatology, Department of Medicine, University of Chicago, 5841 S Maryland, MC-5067, Chicago, IL 60637, USA.

E-mail: vrosic@medicine.bsd.uchicago.edu

This work was delivered as an oral presentation at the Mycosis Fungoides Foundation/International Society for Cutaneous T Cell Lymphoma Symposium at the 66th Annual Meeting of the Society for Investigative Dermatology in St Louis, Missouri in 2005.

Received 21 March 2006; revised 5 May 2006; accepted 8 May 2006; published online 16 June 2006 disease onset in hypopigmented cases is usually in childhood or adolescence; indeed, hypopigmented mycosis fungoides comprises $17 \%$ of all cases of mycosis fungoides in childhood. In contrast to conventional mycosis fungoides, in which the neoplastic cells are CD4 + in the vast majority of cases, ${ }^{5}$ the neoplastic cells in hypopigmented mycosis fungoides have a CD8 + T-cell phenotype.$^{6-9}$ The mechanism of hypomelanosis in hypopigmented mycosis fungoides is as yet unclear. Damaged melanosomes ${ }^{10}$ and a decreased number of melanocytes ${ }^{11,12}$ have been reported in hypopigmented mycosis fungoides lesions.

The etiological basis of hypopigmentation in vitiligo likewise is not completely understood; 
several mechanisms including apoptosis or functional impairment of melanocytes have been proposed. ${ }^{13-20}$ Experimental data support the role of T-cells within active inflammatory lesions of vitiligo in initiating apoptosis of melanocytes. ${ }^{13-15}$ Abnormal interactions between melanocytes and surrounding keratinocytes resulting from a decrease in the melanogenic cytokines produced by the keratinocytes, or from downregulation of specific receptors on the surface of melanocytes have been considered as possible pathways in pathogenesis of hypopigmentation in vitiligo. ${ }^{19,20}$

We hypothesized that as in vitiligo, the pigment loss in hypopigmented mycosis fungoides may be related to alterations in expression of CD117 (also known as stem cell factor receptor or c-KIT), which is present on epidermal melanocytes. To test this hypothesis, we analyzed skin specimens of hypopigmented mycosis fungoides and vitiligo immunohistochemically for CD117 and pan-melanoma antigen cocktail (gp100, tyrosinase, and MART-1) expression.

\section{Materials and methods}

Retrospective case selection was performed by searching the computerized data files of the University of Chicago Dermatopathology Laboratory using commands in the 'diagnosis,' 'clinical notes,' and 'text' fields for 'mycosis fungoides,' 'mycosis fungoides with clinical notes of hypopigmented mycosis fungoides,' and 'decreased/absent melanocytes consistent with vitiligo.' The diagnosis of hypopigmented mycosis fungoides, conventional mycosis fungoides, or vitiligo was made on both clinical and histologic grounds. In cases of conventional mycosis fungoides and hypopigmented mycosis fungoides, patients clinically demonstrating plaque-stage disease and having an unequivocal neoplastic infiltrate in the epidermis on histopathologic examination were selected. Skin margins of cutaneous non-lymphoid tumor resections served as normal skin controls. The study included five cases each of hypopigmented mycosis fungoides, vitiligo, and conventional mycosis fungoides. The study protocol was approved by our IRB. All cases were de-identified and demographic information was not obtained.

Immunohistochemical staining was performed on 4- $\mu$ m-thick, formalin-fixed, paraffin-embedded tissue sections using antibodies against CD3 (Novocastra), CD4 (Neomarkers), CD8 (Neomarkers), pan melanoma cocktail (gp100, tyrosinase, and MART1) (Biocare Medical), and CD117 (DAKO). Slides were deparaffinized in xylene and rehydrated through a graded alcohol series before being placed in $3 \% \mathrm{H}_{2} \mathrm{O}_{2}$ /methanol blocking solution to quench endogenous peroxidase activity, followed by subsequent antigen unmasking. Incubation with the primary antibodies was performed for $1 \mathrm{~h}$ at room temperature at the following dilutions: 1:300 for CD3, 1:20 for CD4, 1:50 for CD8, 1:100 for panmelanoma, and 1:75 for CD117 antibodies. After washing with TBS, the slides were incubated for $30 \mathrm{~min}$ at room temperature with goat anti-mouse or anti-rabbit IgG conjugated to a horseradish peroxidase-labeled polymer (Envision + System, DAKO, Carpinteria, CA, USA), or to an alkaline phosphatase-labeled polymer (MACH3TM, Biocare Medical, Walnut Creek, CA, USA). Reactions were developed with 3, 3'-diaminobenzidine chromogen or Vulcan Red, respectively, and counterstained with hematoxylin. For negative controls, incubation with the primary antibody was omitted, and nonimmune mouse or rabbit serum was substituted.

All the slides were reviewed by two investigators (ZNS and VMP). For pan-melanoma and CD117 immunostains, the immunoreactive cells were scored as number of positive cells per 10 highpower fields (10 fields or entire length of epidermis). For CD3, CD4, and CD8, positive staining was recorded separately in the epidermis and dermis as follows: $1+$ (sparse), $2+$ (moderate), and $3+$ (dense).

\section{Results}

Table 1 shows the CD3/CD4/CD8 staining score in the cases and the controls. The epidermotropic neoplastic cells in all cases of hypopigmented mycosis fungoides had a CD8 + phenotype. Four of the five conventional mycosis fungoides cases had a CD4 + phenotype, and one had a CD8 + phenotype. The infiltrate in both hypopigmented mycosis fungoides and conventional mycosis fungoides was variably dense $(1+$ to $3+)$. The infiltrate was denser in the dermis than the epidermis (hypopigmented mycosis fungoides: $\mathrm{CD} 4=\mathrm{CD} 8$

Table 1 Immunostaining scores for CD3, CD4, and CD8 antibodies

\begin{tabular}{llccccc}
\hline Diagnosis & Marker & Case 1 & Case 2 & Case 3 & Case 4 & Case 5 \\
\hline \multirow{2}{*}{ Vitiligo } & CD3 E/D & $0 / 1$ & $0 / 2$ & $1 / 1$ & $1 / 1$ & $0 / 1$ \\
& CD4 E/D & $0 / 2$ & $0 / 1$ & $0 / 1$ & $0 / 1$ & $0 / 0$ \\
& CD8 E/D & $1 / 1$ & $1 / 3$ & $1 / 1$ & $2 / 3$ & $0 / 1$ \\
HMF & CD3 E/D & $2 / 2$ & $1 / 2$ & $2 / 3$ & $1 / 2$ & $0 / 1$ \\
& CD4 E/D & $1 / 3$ & $1 / 1$ & $1 / 2$ & $0 / 1$ & $0 / 2$ \\
& CD8 E/D & $3 / 3$ & $3 / 2$ & $3 / 3$ & $1 / 2$ & $1 / 1$ \\
CMF & CD3 E/D & $2 / 2$ & $3 / 2$ & $3 / 3$ & $3 / 3$ & $3 / 3$ \\
& CD4 E/D & $2 / 2$ & $2 / 2$ & $0 / 3$ & $2 / 3$ & $3 / 3$ \\
& CD8 E/D & $1 / 2$ & $0 / 1$ & $2 / 3$ & $1 / 2$ & $1 / 2$ \\
Control & CD3 E/D & $0 / 1$ & $0 / 1$ & $0 / 1$ & $0 / 1$ & $0 / 1$ \\
& CD4 E/D & $0 / 1$ & $0 / 1$ & $0 / 1$ & $0 / 1$ & $0 / 1$ \\
& CD8 E/D & $0 / 1$ & $0 / 0$ & $0 / 0$ & $0 / 0$ & $0 / 0$
\end{tabular}

$\mathrm{cMF}=$ conventional mycosis fungoides; $\mathrm{E} / \mathrm{D}=$ epidermal/dermal positivity; $\mathrm{HMF}=$ hypopigmented mycosis fungoides. 


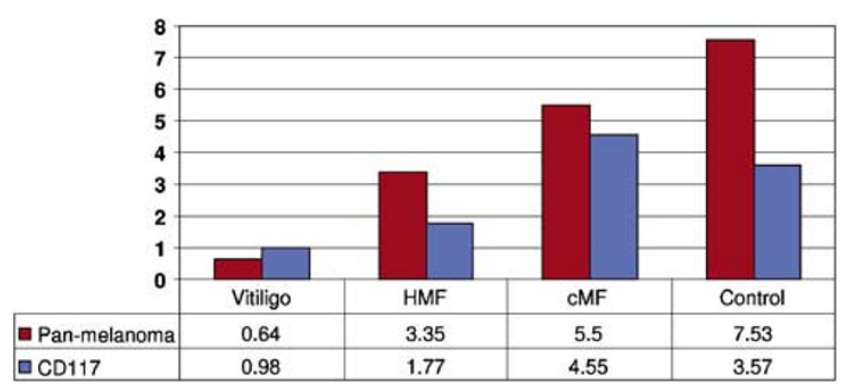

Figure 1 Average immunostaining scores for Pan-melanoma and CD117 antibodies in the cases and controls. Note that the average immunostaining scores for pan-melanoma and CD117 are both lower in vitiligo and in hypopigmented mycosis fungoides as opposed to conventional mycosis fungoides and normal skin controls. $\mathrm{HMF}=$ hypopigmented mycosis fungoides; $\mathrm{cMF}=\mathrm{con}-$ ventional mycosis fungoides.

(three cases), CD4 > CD8 (1), CD4 <CD8 (1); conventional mycosis fungoides: $\mathrm{CD} 4=\mathrm{CD} 8$ ( 2 cases), CD4 > CD8 (3)). In vitiligo, the epidermal infiltrate was sparse and composed of CD8 + cells, while the dermal infiltrate was of mixed CD4 + and CD8 + cells. In normal skin samples, only sparse perivascular infiltration by $\mathrm{CD} 4+$ cells and rare CD8 + cells was noted. The melanosomal (pan-melanoma) antigen score (per high-power field) was as follows: vitiligo: 0-2, mean 0.8; hypopigmented mycosis fungoides: 1-6, mean 3.4; conventional mycosis fungoides: 1-13, mean 5.6; normal skin: 6-8, mean 7.4. The CD117 staining score/hpf was: vitiligo: 0-2, mean 1.2; hypopigmented mycosis fungoides: 0-2, mean 1.6; conventional mycosis fungoides: 2-6, mean 4.4; normal skin: 3-6, mean 3.8. The pan-melanoma and CD117 scores are summarized in Figure 1. Figure 2(a-p) shows the immunostaining pattern of CD4, CD8, pan-melanoma, and CD117 antibodies in cases and control skin.

\section{Discussion}

The etiology and pathogenesis of hypomelanosis in the hypopigmented form of mycosis fungoides has been unclear. A decrease or absence of melanocytes within lesions of hypopigmented mycosis fungoides, akin to melanocyte loss in vitiligo, has been noted leading to speculation on a cytotoxic effect of CD8 + $\mathrm{T}$ cells on melanocytes. ${ }^{1}$ In this context, hypopigmentation during flares of erythrodermic cutaneous T-cell lymphoma has been purported to result from reaction of cytotoxic T-cells. ${ }^{12} \mathrm{On}$ ultrastructural examination, damaged melanosomes $^{10}$ and a decreased number of melanosomes ${ }^{11}$ have been observed in the lesional skin. The pathogenesis of hypopigmentation in vitiligo is also not completely understood; suggested mechanisms include autoimmune, neural, biochemical, oxidative stress, autocytotoxic, viral, and melanocyte detachment. ${ }^{13-20}$ The survival and growth of melanocytes is regulated by binding of stem cell factor (SCF) produced by keratinocytes, to its receptor CD117 on the surface of melanocytes. ${ }^{20}$ Melanocyte-specific microphthalmia-associated transcription factor (MITF-M) expression is regulated downstream of the SCF/SCF receptor linkage ${ }^{21}$ and serves as a transcription factor controlling the expression of tyrosinase mRNA, which essentially leads to melanocyte differentiation. ${ }^{22}$ It is known that CD117 and MITF-M show complex interactions; CD117 signaling modulates MITF-M activity and stability in melanocytes, and MITF-M in turn is needed for the maintenance of CD117 expression on melanoblasts. ${ }^{23}$ Kitamura et $a l^{19}$ observed that the reduction of CD117 and its downstream targets (including MITF-M) by melanocytes might be associated with dysfunction or loss of melanocytes in vitiliginous epidermis. In contrast, Lee et $a l^{20}$ implicated decreased SCF expression by keratinocytes of vitiliginous skin to be responsible for apoptosis and loss of melanocytes. Our findings are in accordance with those of Kitamura et $a l^{19}$ in that there was a decreased expression of the CD117 in the lesional melanocytes of our vitiligo skin specimens. Interestingly, the average number of epidermal CD117-expressing cells (which are melanocytes from their dendritic morphology on immunostains, and positivity for pan-melanoma antigen in immunostaining performed on consecutive serial sections) followed the same pattern of decreased expression in hypopigmented mycosis fungoides as in vitiligo, while the levels in conventional mycosis fungoides were higher and similar to that observed in normal skin. Furthermore, a decreased number of melanocytes per high-power field of the length of the biopsy were present in hypopigmented mycosis fungoides (mean 3.4) and vitiligo (mean 0.8) as compared to 5.6 in conventional mycosis fungoides and 7.4 in normal skin, suggesting a correlation between decreased expression of CD117 and decreased number of melanocytes. Although keratinocyte dysfunction resulting in decreased SCF production is a theoretically possible alternative explanation, such dysfunction has not been reported in mycosis fungoides, and it seems unlikely that both the growth factor and its ligand would be simultaneously affected. In addition, it may be argued that absence of melanogenic antigens (gp100, tyrosinase, and MART-1) is not synonymous with loss of melanocytes and may reflect dysfunction rather than melanocyte loss. Recently a genetic cooperativity has been reported between MITF and Bcl-2 in human melanocytes, and a deficiency of MITF in melanocytes is closely associated with their apoptosis as a result of downregulation of Bcl-2.24 As there is a close link between MITF and CD117 expression, ${ }^{19}$ a decreased expression of CD117 has been associated with markedly decreased expression of MITF-M protein in the lesional epidermis of vitiliginous skin, indicating that melanocytes localized in vitiliginous skin have a high predisposition for apoptosis. In addition, it has been suggested that 

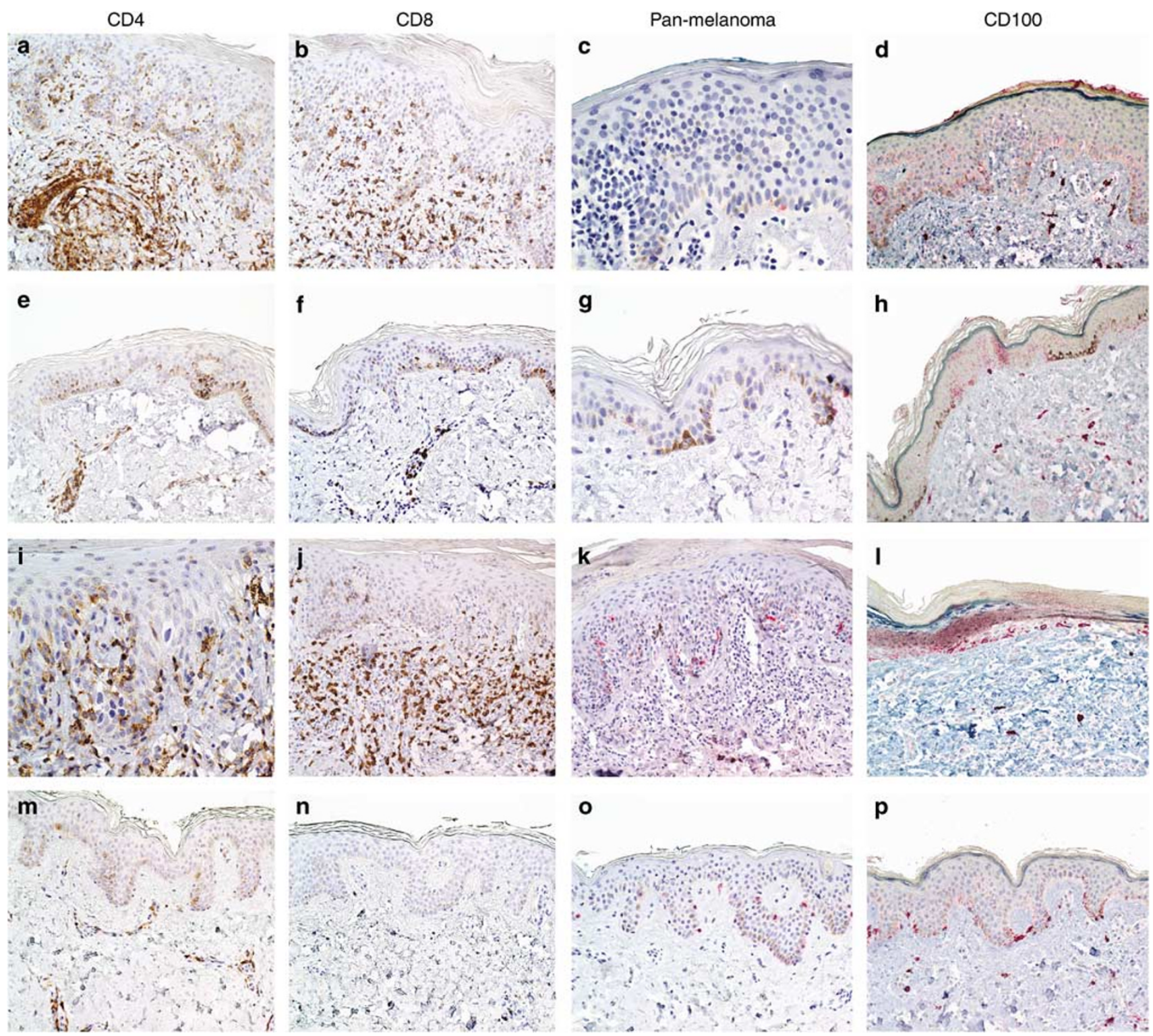

Figure 2 Immunohistochemical staining for CD4, CD8, Pan-melanoma, and CD117 in hypopigmented mycosis fungoides (a-d), vitiligo $(\mathbf{e}-\mathbf{h})$, conventional mycosis fungoides (i-l), and normal skin controls (m-p). Note that the epidermotropic cells in hypopigmented mycosis fungoides are all CD8 positive (b) as opposed to the lymphocytes in conventional mycosis fungoides in which they are CD4 positive (i). Only scattered pan-melanoma and CD117 positive cells are present in hypopigmented mycosis fungoides (c, d), and in vitiligo (g, h), as opposed to conventional mycosis fungoides $(\mathbf{k}, \mathbf{l})$ and normal skin $(\mathbf{o}, \mathbf{p})$.

CD117 serves to maintain the melanocytes within the epidermis by attracting SCF. In the context of this important interaction between CD117 and SCF in stabilizing the melanocytes within the epidermis, it can be inferred from our observations that at least one of the mechanisms of hypomelanosis in hypopigmented mycosis fungoides is likely to be melanocyte loss.

Our study has confirmed earlier observation of the predominance of CD8 + neoplastic $\mathrm{T}$ cells in the epidermis of patients with hypopigmented mycosis fungoides. All cases of hypopigmented mycosis fungoides had a CD8 + immunophenotype. The epidermal lymphocytic infiltrate in vitiligo was also composed of CD8 + cytotoxic T cells whereas the epidermal infiltrate in conventional mycosis fungoides was composed of CD4 $+\mathrm{T}$ cells in four out of five cases; normal skin did not show any notable number of $\mathrm{T}$ cells in the epidermis. Autoantibodies to specific melanosomal surface and cytoplasmic antigens, importantly tyrosinase and tyrosinaserelated proteins (TRP) 1 and 2 have been implicated in melano-cytotoxicity of vitiliginous lesions. ${ }^{25} \mathrm{High}$ frequencies of Melan-A-specific CD8 + T cells have been described in peripheral blood of patients with autoimmune vitiligo. ${ }^{25,26}$ Recent research has shown proliferation of Melan-A/MART 1, gp100, tyrosinase, and TRP-1-specific CD8 $+\mathrm{T}$ cells in in- 
flammatory lesions of spontaneous or vaccinationinduced melanocyte destruction in melanoma patients. ${ }^{27,28}$ By analogy with melanoma-associated and autoimmune vitiligo-associated hypopigmentation, local melanocyte-specific CD8 + T-cell attack could be involved in hypopigmented mycosis fungoides as well, especially in the context of a predominant CD8 + phenotype of the infiltrating lymphocytes in hypopigmented mycosis fungoides expressing the skin homing receptor CLA.

In conclusion, our results offer preliminary support for a possible role of CD8 + T cells and CD117 in the pathogenesis of hypomelanosis in hypopigmented mycosis fungoides, similar to that observed for vitiligo. The comparability in mechanism of hypopigmentation is interesting given the clinical, histopathologic and immunophenotypic similarities between vitiligo and hypopigmented mycosis fungoides. In fact, distinction of vitiligo especially from early stage hypopigmented mycosis fungoides may be difficult; discriminating histopathologic features as suggested in a recent study ${ }^{29}$ may be applied for differential diagnosis in early cases of hypopigmented mycosis fungoides.

We propose that decreased expression of CD117 and its downstream events in melanocytes may be initiated by cytotoxic effects of melanosomalantigen-specific CD8 + neoplastic T-lymphocytes, resulting in destabilization of CD117, and leading to dysfunction and/or loss of melanocytes in hypopigmented mycosis fungoides lesions. Further research involving the expression of downstream effectors of CD117 and the antigen specificity of the clonal CD8 + neoplastic population for specific melanosomal antigens (Melan a/MART 1, tyrosinase and others) will be informative to support our hypothesis.

\section{Acknowledgements}

We are grateful to Dr Deborah Lang for critical review of the manuscript and Dr Christopher Weber for his ingenious handling of the image reproduction.

\section{References}

1 Ardigo M, Borroni G, Muscardin L, et al. Hypopigmented mycosis fungoides in Caucasian patients: a clinicopathologic study of 7 cases. J Am Acad Dermatol 2003;49:264-270.

2 Capizzi R, Rotoli M, Cavalieri S, et al. Hypopigmented mycosis fungoides in a 12-year-old caucasian girl with solely hypopigmented lesions. Dermatology 2003;207: 201-202.

3 Neuhaus IM, Ramos-Caro FA, Hassanein AM. Hypopigmented mycosis fungoides in childhood and adolescence. Pediatr Dermatol 2000;17:403-406.

4 Whitmore SE, Simmons-O'Brien E, Rotter FS. Hypopigmented mycosis fungoides. Arch Dermatol 1994; 130:476-480.
5 Willemze R, Jaffe ES, Burg G, et al. WHO-EORTC classification for cutaneous lymphomas. Blood 2005; 105:3768-3785.

6 Ben-Amitai D, Michael D, Feinmesser M, et al. Juvenile mycosis fungoides diagnosed before 18 years of age. Acta Derm Venerol 2003;83:451-456.

7 El-Shabrawi-Caelen L, Cerroni L, Medeiros LJ, et al. Hypopigmented mycosis fungoides: frequent expression of a CD8+ T-cell phenotype. Am J Surg Pathol 2002;26:450-457.

8 Wain EM, Orchard GE, Whittaker SJ, et al. Outcome in 34 patients with juvenile-onset mycosis fungoides: a clinical, immunophenotypic, and molecular study. Cancer 2003;98:2282-2290.

9 Peters MS, Thibodeau SN, White Jr JW, et al. Mycosis fungoides in children and adolescents. J Am Acad Dermatol 1990;22:1011-1018.

10 Breathnach SM, McKee PH, Smith NP. Hypopigmented mycosis fungoides: report of five cases with ultrastructural observations. $\mathrm{Br} \mathrm{J}$ Dermatol 1982;106: 643-649.

11 Goldberg DJ, Schinella RS, Kechijian P. Hypopigmented mycosis fungoides. Speculations about the mechanism of hypopigmentation. Am J Dermatopathol 1986;8:326-330.

12 Bouloc A, Grange F, Delfau-Larue $\mathrm{MH}$, et al. Leucoderma associated with flares of erythrodermic cutaneous T-cell lymphomas: four cases. The French Study Group of Cutaneous Lymphomas. Br J Dermatol 2000; 143:832-836.

13 Steitz J, Wenzel J, Gaffal E, et al. Initiation and regulation of $\mathrm{CD} 8+\mathrm{T}$ cells recognizing melanocytic antigens in the epidermis: implications for the pathophysiology of vitiligo. Eur J Cell Biol 2004;83:797-803.

14 Mandelcorn-Monson RL, Shear NH, Yau E, et al. Cytotoxic T lymphocyte reactivity to gp100, MelanA/ MART-1, and tyrosinase, in HLA-A2-positive vitiligo patients. J Invest Dermatol 2003;121:550-556.

15 van Den Wijngaard R, Wankowicz-Kalinska A, Pals S, et al. Autoimmune melanocyte destruction in vitiligo. Lab Invest 2001;81:1061-1067.

16 Ongenae K, Van Geel N, Naeyaert JM. Evidence for an autoimmune pathogenesis of vitiligo. Pigment Cell Res 2003;16:90-100.

17 Gauthier Y, Andre MC, Taieb A. A critical appraisal of vitiligo etiologic theories: is melanocyte loss a melanocytorrhagy? Pigment Cell Res 2003;16:322-332.

18 Grimes PE, Sevall JS, Vojdani A. Cytomegalovirus DNA identified in skin biopsy specimens of patients with vitiligo. J Am Acad Dermatol 1996;35:21-26.

19 Kitamura R, Tsukamoto K, Harada K, et al. Mechanisms underlying the dysfunction of melanocytes in vitiligo epidermis: role of SCF/KIT protein interactions and the downstream effector, MITF-M. J Pathol 2004; 202:463-475.

20 Lee AY, Kim NH, Choi WI, et al. Less keratinocytederived factors related to more keratinocyte apoptosis in depigmented than normally pigmented suctionblistered epidermis may cause passive melanocyte death in vitiligo. J Invest Dermatol 2005;124:976-983.

21 Hemesath TJ, Price ER, Takemoto C, et al. MAP kinase links the transcription factor Microphthalmia to C-Kit signaling in melanocytes. Nature 1998;391:298-301.

22 Tachibana M, Takeda K, Nobukuni Y, et al. Ectopic expression of MITF, a gene for Waardenburg syndrome type 2, converts fibroblasts to cells with melanocyte characteristics. Nat Genet 1996;14:50-54. 
23 Hou L, Panthier JJ, Arnheiter H. Signaling and transcriptional regulation in the neural crest-derived melanocyte lineage: interactions between KIT and MITF. Development 2000;127:5379-5389.

24 McGill GG, Horstmann M, Widlund HR, et al. Bcl2 regulation by the melanocyte master regulator Mitf modulates lineage survival and melanoma cell viability. Cell 2002;109:707-718.

25 Palermo B, Campanelli R, Garbelli S, et al. Specific cytotoxic T lymphocyte responses against Melan-A/ MART1, tyrosinase and gp100 in vitiligo by the use of major histocompatibility complex/peptide tetramers: the role of cellular immunity in the etiopathogenesis of vitiligo. J Invest Dermatol 2001;117: 326-332.
26 Ogg GS, Rod Dunbar P, Romero P, et al. High frequency of skin-homing melanocyte-specific cytotoxic T lymphocytes in autoimmune vitiligo. J Exp Med 1998;188: 1203-1208.

27 Becker JC, Guldberg P, Zeuthen J, et al. Accumulation of identical $\mathrm{T}$ cells in melanoma and vitiligo-like leukoderma. J Invest Dermatol 1999;113:1033-1038.

28 Yee C, Thompson JA, Roche P, et al. Melanocyte destruction after antigen-specific immunotherapy of melanoma: direct evidence of T-cell-mediated vitiligo. J Exp Med 2000;192:1637-1644.

29 El-Darouti MA, Marzouk SA, Azzam O, et al. Vitiligo vs hypopigmented mycosis fungoides (histopathological and immunohistochemical study, univariate analysis). Eur J Dermatol 2006;16:17-22. 